\title{
Three-wire thermocouple: Frequency response in constant flow
}

L. J. Forney

School of Chemical Engineering, Georgia Tech, Atlanta, Georgia 30332

G. C. Fralick

NASA-Lewis Research Center, Cleveland, Ohio 44135

(Received 20 September 1994; accepted for publication 13 January 1995)

Theory and experimental measurements are compared with a novel three-wire thermocouple. Signals from three wires of unequal diameters are recorded from the thermocouple suspended in constant flow with a periodic temperature fluctuation. It is demonstrated that the reconstructed signal from the three-wire thermocouple requires no compensation for $\omega \leqslant 5 \omega_{1}$, where $\omega_{1}$ is the natural frequency of the smaller wire. The latter result represents a significant improvement compared to previous work with two-wire thermocouples. A correction factor has also been derived to account for wires of arbitrary diameter. (C) 1995 American Institute of Physics.

\section{INTRODUCTION}

The evaluation of jet engine performance and fundamental studies of combustion phenomena depend on the measurement of turbulent fluctuating temperatures of the gas within the engine. Historically, these temperatures have been measured with thermocouples. ${ }^{1}$ The advantages of thermocouples are their low cost, reliability, and simplicity since they do not require optical access or elaborate support electronics. However, the design of a thermocouple represents a compromise between accuracy, ruggedness, and rapidity of response.

For example, the measurement of fluctuating temperatures in the high-speed exhaust of a gas turbine engine combustor is required to characterize the local gas density gradients or convective heat transfer. ${ }^{2}$ Although thermocouples are suitable for the measurement of high-frequency temperature fluctuations $(<1 \mathrm{kHz})$ in a flowing gas or liquid, the measured signal must be compensated since the frequency of the time-dependent fluid temperature is normally much higher than the natural frequency of the thermocouple probe. ${ }^{3}$ Moreover, use of a single-wire thermocouple in constant velocity flows requires knowledge of the fluid velocity and properties (e.g., viscosity, density, etc.) to determine the natural frequency.

The present paper describes the performance of a novel three-wire thermocouple of unequal diameters that does not require compensation at lower fluid temperature frequencies nor any knowledge of the fluid velocity or properties. ${ }^{4,5}$ The results of experimental measurements are presented along with the suggested procedure for the reduction of the data from the three-wire thermocouple as shown in Fig. 1.

\section{THEORY}

Use of a single-wire thermocouple requires knowledge of the fluid velocity and properties to determine the natural frequency. The latter quantity is necessary to establish a frequency dependent compensation factor for the measured signal.

\section{A. Natural frequency of single wire}

The conservation of energy for a single wire is

$$
\frac{d T}{d t}=\omega_{n}\left(T_{g}-T\right),
$$

where the natural frequency

$$
\omega_{n}=\frac{4 h}{\rho c D} .
$$

Here $\omega_{n}$ is the natural frequency $\left(\mathrm{s}^{-1}\right), T$ the temperature of the thermocouple (K), $T_{g}$ the temperature of the gas $(K), t$ the time (s), $\rho$ the wire density $\left(\mathrm{kg} / \mathrm{m}^{3}\right), c$ the heat capacity of wire $(\mathrm{J} / \mathrm{kg} \mathrm{K}), h$ the heat transfer coefficient $\left(\mathrm{W} / \mathrm{m}^{2} \mathrm{~K}\right)$, and $D$ the wire diameter $(\mathrm{m})$.

It is convenient to rewrite the heat transfer coefficient in terms of the Nusselt number

$$
h=\frac{k_{g}}{D} \mathrm{Nu},
$$

where $k_{g}$ is the thermal conductivity of the gas $(\mathrm{W} / \mathrm{m} \mathrm{K})$ and $\mathrm{Nu}$ the Nusselt number. Thus the natural frequency in Eq. (2) becomes

$$
\omega_{n}=\frac{4 k_{g} \mathrm{Nu}}{(\rho c) D^{2}},
$$

where $^{6}$

$$
\mathrm{Nu}=C_{0} \operatorname{Re}^{m} \operatorname{Pr}^{1 / 3}
$$

and

$$
\begin{aligned}
& \operatorname{Re}=\frac{V D}{v_{g}}, \\
& \operatorname{Pr}=\frac{\mu c_{p}}{k_{g}} .
\end{aligned}
$$

Here, $\operatorname{Re}$ and $\operatorname{Pr}$ are the Reynolds and Prandtl numbers, respectively, and $\mu$ is the viscosity of gas $(\mathrm{kg} / \mathrm{m} \mathrm{s}), v_{g}$ the kinematic viscosity of gas $\left(\mathrm{m}^{2} \mathrm{~s}^{-1}\right)$, and $V$ the velocity of gas $(\mathrm{m} / \mathrm{s})$. 


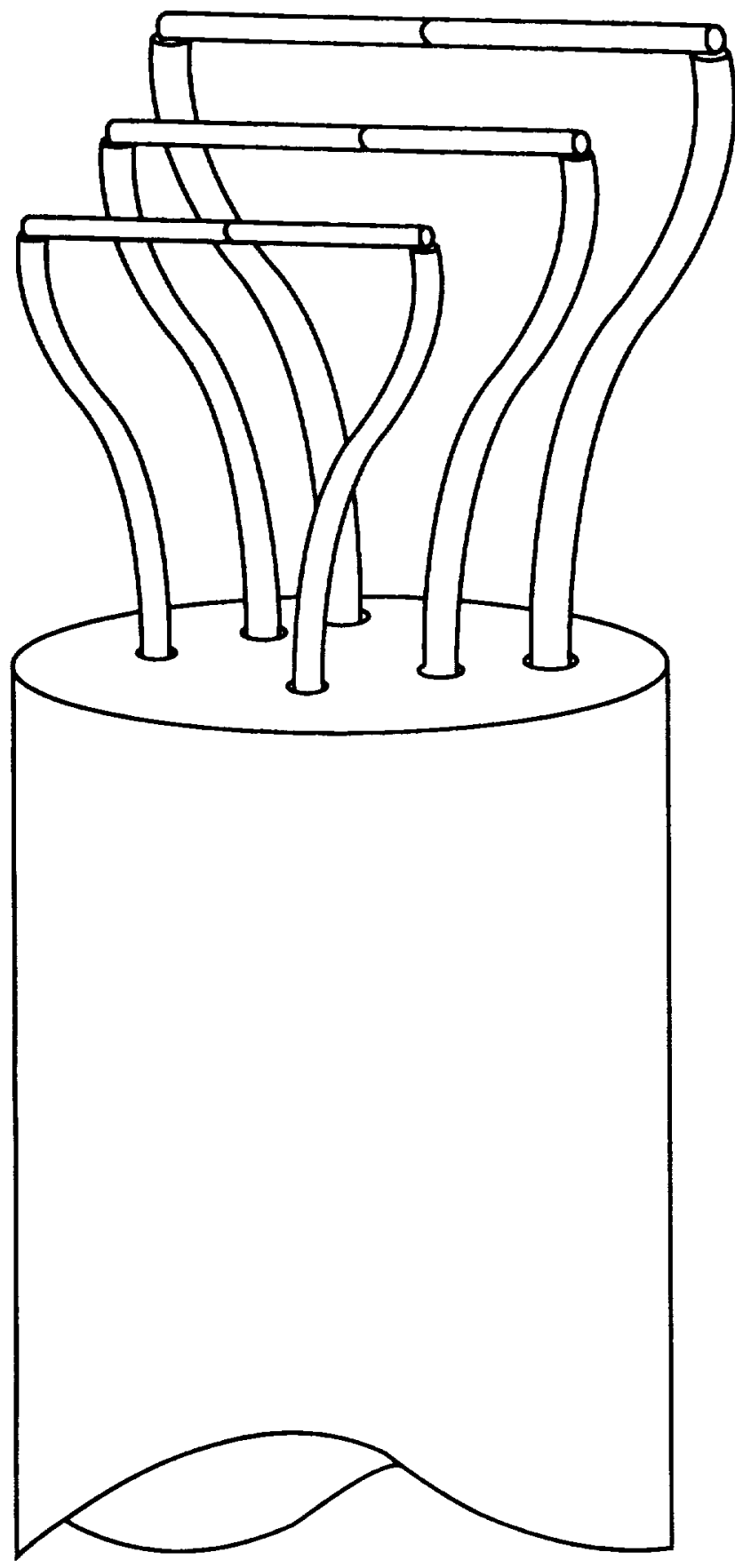

FIG. 1. Schematic of three-wire thermocouple.

The natural frequency of a single wire from Eqs. (4) and (5) can now be written in the form

$$
\omega_{n}=\frac{k_{g} C_{0} 4 \operatorname{Re}^{m} \operatorname{Pr}^{1 / 3}}{(\rho c) D^{2}}
$$

or separating the wire diameter

$$
\omega_{n}=C D^{m-2}
$$

where the coefficient $C$ depends on both fluid and wire parameters, or

$$
C=\frac{4 k_{g} C_{0} V^{m} \operatorname{Pr}^{1 / 3}}{(\rho c) v_{g}^{m}}
$$

Substituting values corresponding to a type-K thermocouple and a gas velocity $V=25 \mathrm{~m} / \mathrm{s}$ for air at standard conditions into Eqs. (7) and (8), one obtains values of the natural frequency:

\begin{tabular}{rrc}
\hline \hline$\omega_{n}\left(\mathrm{~s}^{-1}\right)$ & $D(\mathrm{~m})$ & $D(\mathrm{mil})$ \\
\hline 52.6 & $50.8 \times 10^{-6}$ & 2 \\
18.3 & $101.6 \times 10^{-6}$ & 4 \\
6.5 & $203.2 \times 10^{-6}$ & 8 \\
\hline
\end{tabular}

\section{B. Determination of gas temperature}

The use of a single-wire thermocouple requires knowledge of the fluid velocity and additional properties such as viscosity or density. There are three unknowns that appear in Eqs. (1) and (7): (a) the coefficient $C$ given by Eq. (8), (b) $m$, the exponent of the wire Reynolds number in Eqs. (6) $-(8)$, and (c) the gas temperature $T_{g}$.

Consider three thermocouples of unequal diameters $D_{1}<D_{2}<D_{3}$ as shown in Fig. 1. The conservation of energy equation for each wire reduces to

$$
\begin{aligned}
& \frac{d T_{1}}{d t}=C D_{1}^{m-2}\left(T_{g}-T_{1}\right), \\
& \frac{d T_{2}}{d t}=C D_{2}^{m-2}\left(T_{g}-T_{2}\right), \\
& \frac{d T_{3}}{d t}=C D_{3}^{m-2}\left(T_{g}-T_{3}\right) .
\end{aligned}
$$

In principle it is possible to solve for the three unknowns that appear in Eqs. (9)-(11) in terms of the instantaneous values of the wire temperatures $T_{1}, T_{2}$, and $T_{3}$ and their derivatives. However, because of the empirical nature of the Nusselt number given in Eq. (5) and uncertainty in the wire diameters near the thermocouple junctions, we choose to eliminate the coefficient $C$ and solve for the gas temperature $T_{g}$ from Eqs. (9)-(11). Moreover, because the conservation of energy equations are linear at constant ambient velocities, we choose to use transformed data profiles.

Thus taking the fast Fourier transform (FFT) of Eqs. (9)-(11), squaring Eq. (10), and dividing by the product of Eqs. (9) and (11), one obtains

$$
\left(\frac{\bar{T}_{2}^{2}}{\bar{T}_{1} \bar{T}_{3}}\right)=\left(\frac{D_{2}^{2}}{D_{1} D_{3}}\right)^{m-2} \frac{\left(\bar{T}_{g}-\bar{T}_{2}\right)^{2}}{\left(\bar{T}_{g}-\bar{T}_{1}\right)\left(\bar{T}_{g}-\bar{T}_{3}\right)}
$$

Substituting

$$
r=\bar{T}_{2}^{2} / \bar{T}_{3} \bar{T}_{1}
$$

into Eq. (12) and anticipating that the wire diameter ratio $D_{2}^{2} / D_{1} D_{3} \sim O(1)$ or

$$
\left(\frac{D_{2}^{2}}{D_{1} D_{3}}\right)^{m-2}=1+\epsilon \text {, }
$$


the transform of the gas temperature becomes

$$
\bar{T}_{g}=\frac{-b_{0}-\sqrt{b_{0}^{2}-4 a_{0} c_{0}}}{2 a_{0}} .
$$

Here, $b_{0}=b+2 \epsilon \bar{T}_{2}+O(\epsilon)^{2},-4 a_{0} c_{0}=4 \epsilon \bar{T}_{2}^{2}(r-1)$ $+O(\epsilon)^{2}, b_{0}^{2}-4 a_{0} c_{0}=b^{2}+\epsilon \gamma+O(\epsilon)^{2}$, and $a_{0}=r-1-\epsilon$, where

$$
b=2 \bar{T}_{2}-r\left(\bar{T}_{3}+\bar{T}_{1}\right)
$$

and

$$
\gamma=4 T_{2}\left[b+\bar{T}_{2}(r-1)\right] .
$$

We now seek a Taylor-series expansion of Eq. (15) for the transformed gas temperature about the point $\epsilon=0$. Thus to first order in $\epsilon$ where

$$
f(\epsilon)=\sqrt{b_{0}^{2}-4 a_{0} c_{0}}=\sqrt{b^{2}+\epsilon \gamma}
$$

and

$$
g(\epsilon)=\frac{1}{a_{0}}=\frac{1}{r-1-\epsilon},
$$

one obtains

$$
f(\epsilon)=b+\frac{\gamma}{2 b} \epsilon+O(\epsilon)^{2}
$$

and

$$
g(\epsilon)=\frac{1}{a}+\frac{\epsilon}{a^{2}}+O(\epsilon)^{2},
$$

where

$$
a=r-1 .
$$

Substituting Eqs. (17) and (18) into Eq. (15) and retaining terms $O(\epsilon)$, one obtains

$$
\bar{T}_{g}=-\frac{b}{a}-\epsilon\left(\frac{2 \bar{T}_{2}}{a}+\frac{\bar{T}_{2}^{2}}{b}+\frac{b}{a^{2}}\right),
$$

where $a$ and $b$ are defined by Eqs. (13), (19), and (16), respectively.

We now define the transform of the gas temperature for the particular case where the wire diameter ratio $D_{2}^{2} / D_{1} D_{3}=1$ in the form

$$
\bar{T}_{g}(0)=-\frac{b}{a}
$$

or

$$
\bar{T}_{g}(0)=\frac{\bar{T}_{2}\left(\bar{T}_{2} \bar{T}_{1}+\bar{T}_{2} \bar{T}_{3}-2 \bar{T}_{1} \bar{T}_{3}\right)}{\left(\bar{T}_{2}^{2}-\bar{T}_{1} \bar{T}_{3}\right)} .
$$

Substituting for the quantity $b$ defined by Eq. (21) into Eq. (20), one obtains the final result

$$
\bar{T}_{g}(\epsilon)=\bar{T}_{g}(0)+\epsilon\left(\frac{1}{a \bar{T}_{g}(0)}\right)\left[\bar{T}_{2}-\bar{T}_{g}(0)\right]^{2}
$$

where

$$
a=\frac{\bar{T}_{2}^{2}}{\bar{T}_{1} \bar{T}_{3}}-1
$$

TABLE I. Wire diameter combinations $(m=1 / 2)$.

\begin{tabular}{cccc}
\hline \hline$D_{1}$ (mil) & $D_{2}$ (mil) & $D_{3}$ (mil) & $\epsilon$ \\
\hline 2 & 4 & 8 & 0.0 \\
2 & 3 & 5 & 0.17 \\
2 & 3 & 4 & -0.16 \\
\hline \hline
\end{tabular}

and $\bar{T}_{g}(0)$ is defined by Eq. (22). The inverse transform of $\bar{T}_{8}(\epsilon)$ becomes the reconstructed gas temperature or

$$
T_{g}=\mathrm{FFT}^{-1}\left[\bar{T}_{g}(\epsilon)\right]
$$

for assumed values of $\epsilon$.

It is useful to consider the effects of wire diameter on the magnitude of the perturbation parameter $\epsilon$. In general it is desirable to have small diameter wires so that their natural frequencies are shifted to larger values. Listed in Table I are three possible wire combinations that are commercially available along with the magnitude of $\epsilon$. The values of $\epsilon$ are computed from Eq. (14) assuming that the wire diameters are exactly as listed by the manufacturer, which is very unlikely in practice. For example, a typical value of the exponent $m=1 / 2$ in Eq. (14) suggests that a $7 \%$ error in $D_{2}$ would yield a value of $\epsilon \leqslant 0.2$ for the wire diameters listed in the first row of Table I.

\section{EXPERIMENT}

\section{A. Apparatus}

In the present experiment, thermocouple sensors are exposed to a constant velocity airstream $(<25 \mathrm{~m} / \mathrm{s})$ of varying temperature. In particular, the dynamic response of the thermocouple is measured for a periodic temperature profile of varying frequency. A rotating wheel configuration is used to deliver the test airstream to the proposed sensors. A similar experimental apparatus was described in detail by Elmore et $a l^{7}$ and Forney et $a l^{8}$

A schematic of the rotating wheel apparatus used in the present experiment is shown in Fig. 2. As the wheel rotates, holes pass the two air supply tubes (3/4-in. i.d. copper) that allow slugs of hot $\left(\sim 55^{\circ} \mathrm{C}\right)$ and cold $\left(\sim 30^{\circ} \mathrm{C}\right)$ air to alternately enter a transition tube assembly mounted directly above the rotating wheel. In the transition tube the slugs of hot and cold air coalesce into a single airstream providing a periodic temperature profile covering a range of gas temperature frequencies from roughly 1 to $60 \mathrm{~Hz}$. A 3/4-1/2 in. smooth copper adapter was inserted at the end of the coalescing tube (location of thermocouple) in Fig. 2 to increase the air velocity to $25 \mathrm{~m} / \mathrm{s}$.

The analog temperature signal is digitized with a Data Translation DT-2801 A/D board mounted in an expansion slot of an IBM AT compatible computer as shown in Fig. 2. ASYST software was loaded onto the hard disk of the personal computer and this provided a flexible system for data storage, manipulation, and display. The true temperature profile of the airstream is measured with a constant current anemometer (TSI 1054-A) and sensor (1210-T1.5).

The three type-K thermocouple wires were of diameter $D_{1}=50.8,(2 \mathrm{mils}), D_{2}=101.6$, and $D_{3}=203.2 \mu \mathrm{m}$ as 


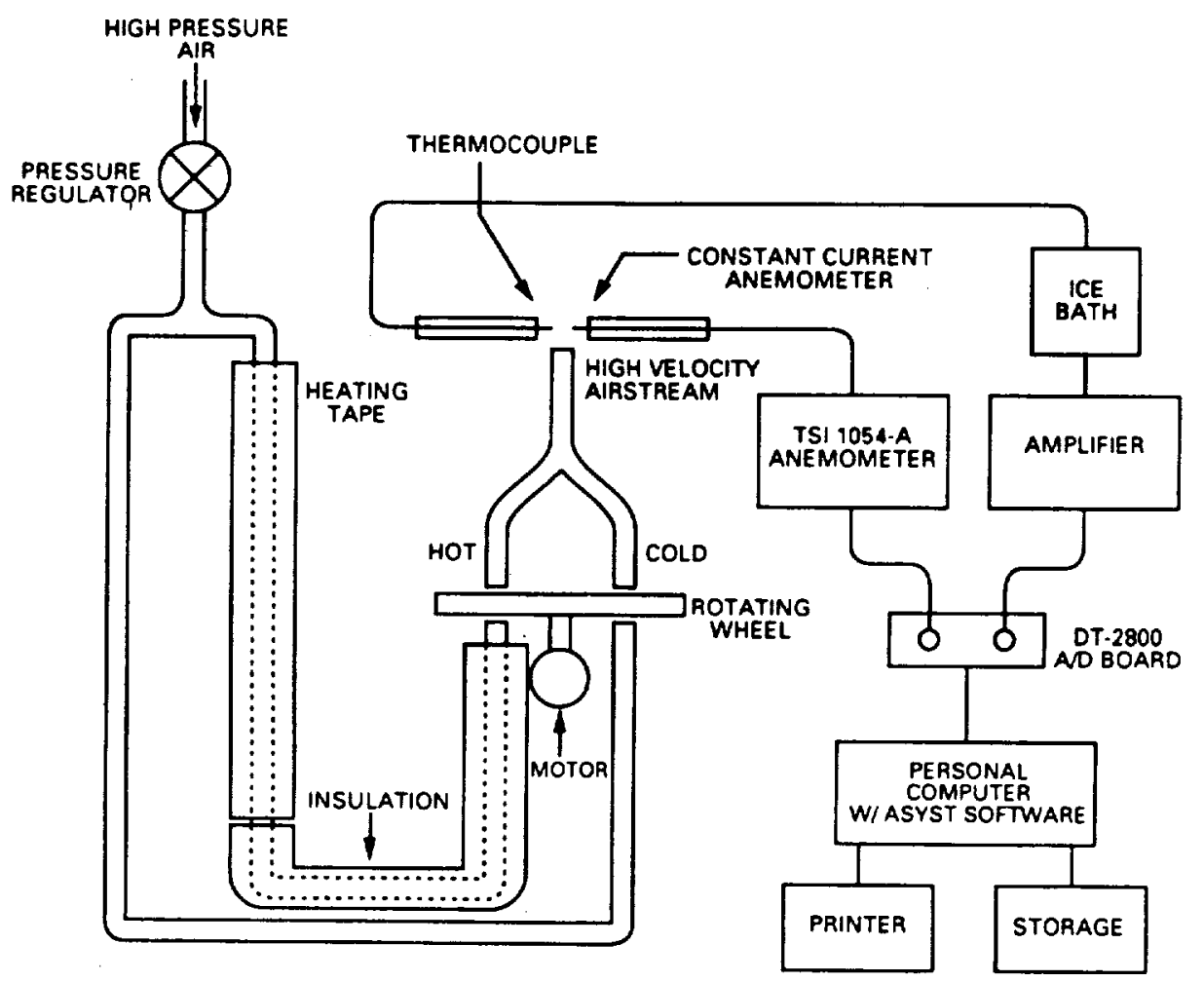

FIG. 2. Schematic of rotating wheel apparatus.

shown in Fig. 1. The thermocouple junctions were fabricated by the Research Instrumentation Branch at the NASA Lewis Center. The wires were cut with a razor blade to produce a flat edge perpendicular to the wire axis. The wire segments were then mounted in a fixture such that the two faces of the junction were held together by springs. The junction was then formed by laser heating. The laser used was a KORAD model KWD Nd:YAG laser, operating at a wavelength of $1060 \mathrm{~nm}$. The power settings depend on the wire size and material, but for the thermocouples described in this report, a pulse duration of $4 \mathrm{~ms}$ was used, delivering a total energy of approximately $2 \mathrm{~J}$.

\section{B. Procedure}

ASYST software was developed to acquire temperature data sequentially from the thermocouples and constant current anemometer. The data are digitized for four channels at a sampling rate of $512 \mathrm{~Hz}$ per channel for a total sample time of $0.5 \mathrm{~s}$. After data acquisition, the FFT is taken for each channel. The largest peak (first harmonic) in the amplitude of the FFT is located for each channel which provides the frequency of the temperature profile. The ASYST software also records the amplitude ratio of the first harmonic for the $\mathbf{5 0 . 8}$ $\mu \mathrm{m}$ thermocouple-to-anemometer output. The amplitude ratio was recorded for each frequency setting of the rotating wheel and provided a value for the natural frequency $\omega_{1}$ of the small wire.

The FFT of the signal from each thermocouple channel was substituted into Eq. (19) by the ASYST code along with an estimate of the parameter $\epsilon$. The inverse transform of $\bar{T}_{g}$ by the ASYST code provided a reconstruction of the true gas temperature.

\section{RESULTS AND DISCUSSION}

The temperature profiles for the ambient gas (large amplitude) and the three type-K thermocouple wires are shown in Fig. 3. The three wire diameters used in the present study are $D_{1}=50.8$, (2 mils), $D_{2}=101.6$, and $D_{3}=203.2 \mu \mathrm{m}$. The angular frequency of the gas temperature in Fig. 3 is $\omega=86 \mathrm{~s}^{-1}(\sim 14 \mathrm{~Hz})$ while the natural frequency of the small

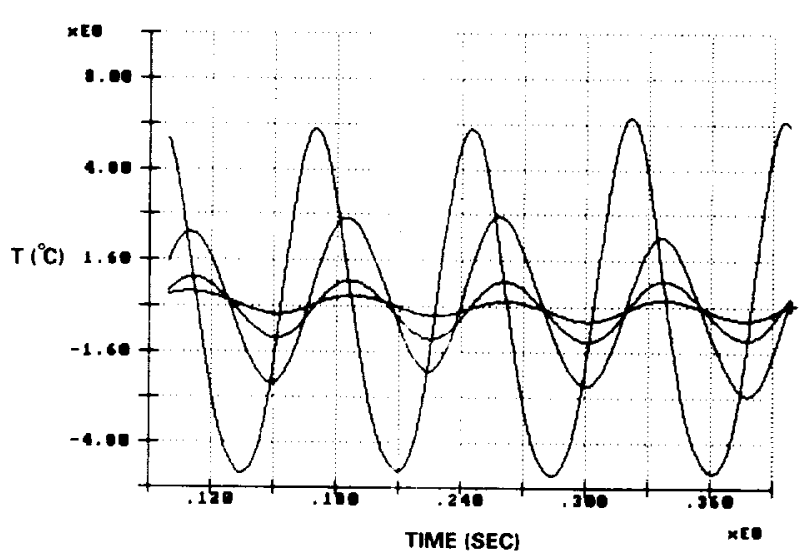

FIG. 3. Temperature profiles for the gas (large amplitude) and three type-K thermocouple wires. Three wires: $D_{1}=50.8 \mu \mathrm{m}, \omega_{1}=42 \mathrm{~s}^{-1}, D_{2}=101.6$ $\mu \mathrm{m}, D_{3}=203.2 \mu \mathrm{m}$. Gas temperature frequency: $\omega=86 \mathrm{~s}^{-1}$. 


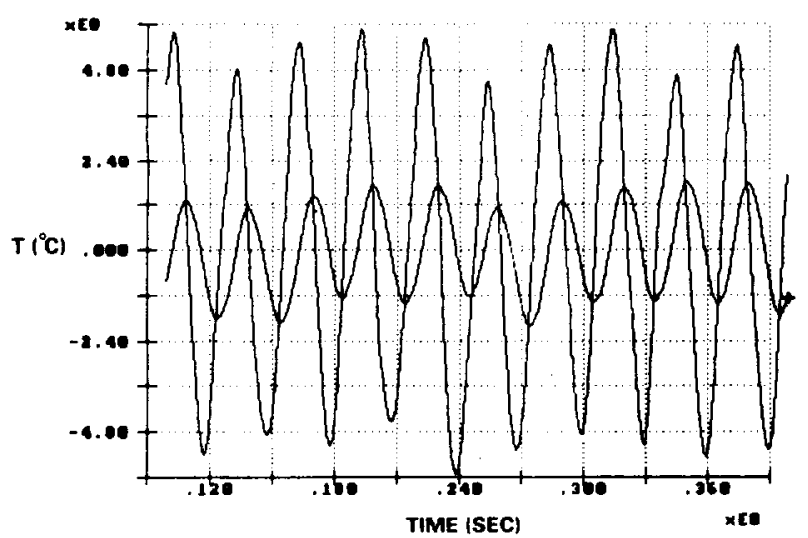

FIG. 4. Gas temperature profile compared to reconstructed temperature derived from three-wire thermocouple. $\omega_{1}=61 \mathrm{~s}^{-1}, \omega=209 \mathrm{~s}^{-1}, \epsilon=-0.2$.

wire $\omega_{1}=42 \mathrm{~s}^{-1}$. The natural frequency of the small wire corresponds to an ambient gas velocity of roughly $10 \mathrm{~m} / \mathrm{s}$.

Figures 4, 5, and 6 illustrate the effects of increasing the magnitude of the wire diameter parameter $\epsilon$ defined by Eq. (14). The small phase angle between the large ambient gas temperature profiles that appear in Figs. 4 and 5 and the smaller temperature profiles reconstructed from the thermocouple probe allows one to distinguish between both profiles in Fig. 6. In the latter case, the temperature profile on the left is that of the ambient gas while the profile on the right was determined by taking the inverse FFT of $\bar{T}_{g}(\epsilon)$ defined by Eq. (23) with $\epsilon=0.2$. In all three figures the natural frequency of the small wire $\omega_{1}=61 \mathrm{~s}^{-1}\left(V \approx 20 \mathrm{~ms}^{-1}\right)$ while the angular frequency of the ambient gas temperature is $\omega=209$ $\mathrm{s}^{-1}(\sim 33 \mathrm{~Hz})$.

It is clear that a value of the parameter $\epsilon=0.2$ provides a signal that requires no compensation for the frequency ratio $\omega / \omega_{1}=3.4$. It should also be noted that a $7 \%$ error in the diameter of $D_{2}$ would account for a value of $\epsilon=0.2$. Further, the coefficient of $\epsilon$ in Eq. (23) depends on the difference between the uncompensated FFT of the signal from the wire of diameter $D_{2}$ and the compensated FFT of the signal from the three-wire probe with a particular diameter ratio

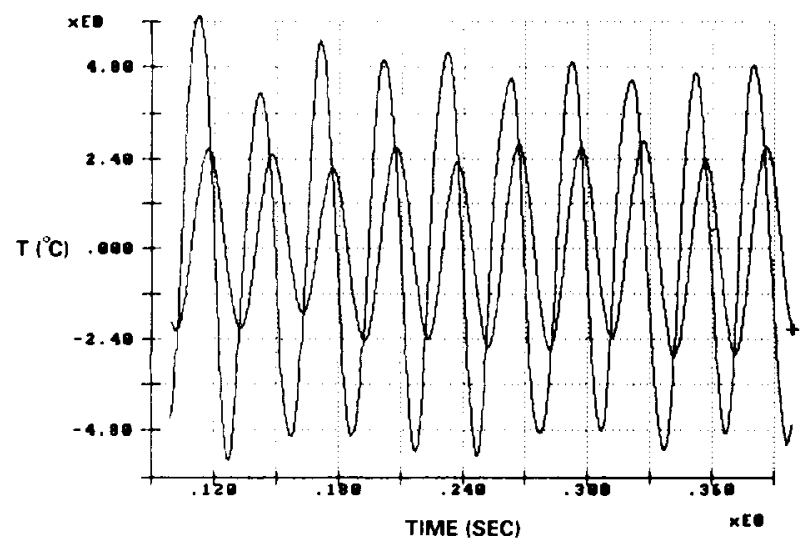

FIG. 5. Same conditions as Fig. 4. $\epsilon=0.0$

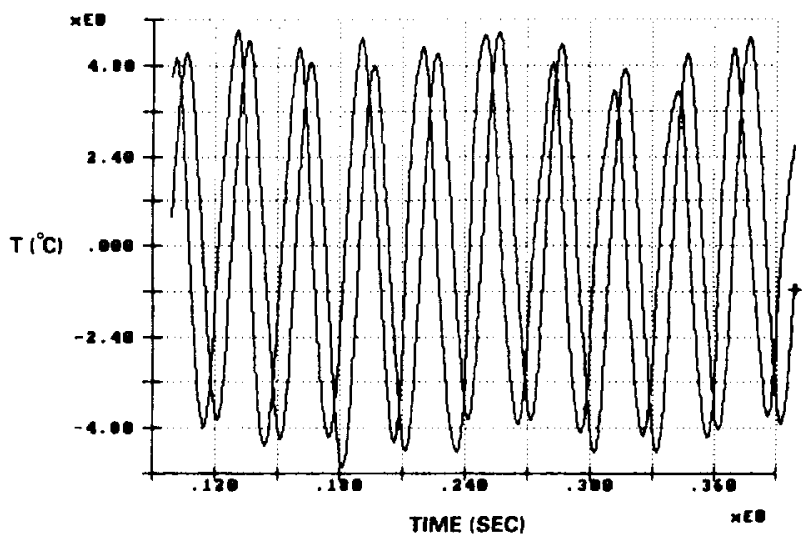

FIG. 6. Same conditions as Fig. 4. $\epsilon=0.2$.

$D_{2}^{2}=D_{1} D_{3}(\epsilon=0)$. Thus the contribution to the signal by the second term on the right of $\mathrm{Eq}$. (23) is negligible at low frequencies but increases with larger values of $\omega / \omega_{1}$.

The amplitude ratio of the three-wire thermocouple output-to-true gas temperature as a function of gas temperature frequency has been plotted in Fig. 7. Also shown is the first-order response of the small wire. The open symbols correspond to a value of $\epsilon=0$ in Eq. (23) where the amplitude ratio requires no compensation for $\omega \leqslant 2 \omega_{1}$. For the range of values $2 \omega_{1}<\omega<5 \omega_{1}$ the amplitude ratio follows the function

$$
A=1.92\left(\frac{\omega}{\omega_{1}}\right)^{-1.03}
$$

It should be noted that the first-order response is also proportional to $\left(\omega / \omega_{1}\right)^{-1}$ at large frequencies.

A significant improvement is observed, however, when the parameter $\epsilon=0.2$ as indicated by the solid symbols in Fig. 7. For the latter case, no compensation is required for

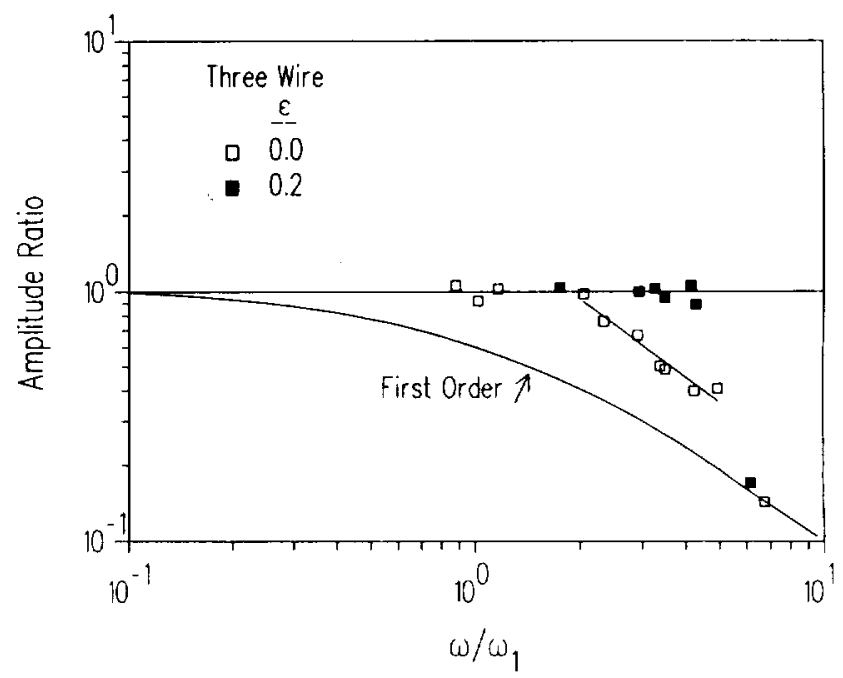

FIG. 7. Amplitude ratio of three-wire thermocouple output-to-true gas temperature. Also shown is first-order response of small wire 
$\omega \leqslant 5 \omega_{1}$. As discussed earlier, the amplitude ratio data coelesce at low gas temperature frequencies where the coefficient of $\epsilon$ in Eq. (23) approaches zero.

Both values of $\epsilon=0.0$ or 0.2 are of little value, however, when $\omega \geqslant 6 \omega_{1}$ where the amplitude ratio for the three-wire thermocouple reduces to the first-order response of the smaller wire. The latter result appears to occur at a gas frequency of $\sim 60 \mathrm{~Hz}$. For the present experimental system the signal-to-noise ratio may be responsible for the small response at large frequencies. This situation may be improved by replacing the two larger wires of 4 and 8 mils diameter by wires of 3 and 4 mils, respectively, as listed in Table I.

\section{ACKNOWLEDGMENTS}

The work reported herein was sponsored by a NASA cooperative agreement No. NCC 3-302. The authors also wish to acknowledge the support of Dr. W. D. Williams, head of the Research Sensor Technology Branch of NASA-Lewis.

${ }^{1}$ R. R. Dils and P. S. Follansbee, Instrumentation in the Aenospace Industry, edited by B. Washburn (ISA, 1976), Vol. 21.

${ }^{2}$ G. C. Fralick, NASA Report No. CP-2465, 1985, pp. 81-85.

${ }^{3}$ M. D. Scadron and I. Warshawsky, NACA Report No. TN-2599, 1952.

${ }^{4}$ G. C. Fralick, Patent Disclosure, April 25, 1990.

${ }^{5}$ L. J. Forney and G. C. Fralick, Rev. Sci. Instrum. 65, 3252 (1994).

${ }^{6}$ F. P. Incropera and D. P. DeWitt, Fundamentals of Heat and Mass Transfer, 2nd ed. (Wiley, New York, 1985).

${ }^{7}$ D. L. Elmore, W. W. Robinson, and W. B. Watkins, NASA Report No. CR-179513, 1986.

${ }^{8}$ L. J. Forney, E. L. Meeks, J. Ma, and G. C. Fralick, Rev. Sci. Instrum. 64, 3236 (1993). 

